\title{
Exact ferromagnetic ground state of pentagon chains
}

\author{
Miklos Gulácsi, György Kovács, and Zsolt Gulácsi \\ Department of Theoretical Physics, University of Debrecen, H-4010 Debrecen, Hungary
}

(Dated: June 21, 2014)

\begin{abstract}
We model conducting pentagon chains with a multi orbital Hubbard model and prove that well below half filling exact ferromagnetic ground states appear. The rigorous method we use is based on the transformation of original hamiltonian into positive semidefinite form. This technique is independent of the spatial dimesion and does not require integrability of the model. The obtained ferromagnetism is connected to dispersionless bands but in a much broader sense than flat band ferromagnetism requires, where on every site a Hubbard term is present. In our case only a small percentage of, even randomly distributed, sites are only interacting.
\end{abstract}


Recent observation of ferromagnetism in polythiophene compounds $\frac{1}{2}$ has generated a widespan interest and a heightened research effort to develop plastic ferromagnets and more generally to understand ferromagnetism in systems made entirely of nonmanetic elements. As a result several theories have emerged to describe ferromagnetism in these systems ${ }^{2}$, with particular focus on ferromagnetism due to dispersionless bands ${ }^{3} \underline{4}$ or periodic Anderson model ${ }^{5}$.

These theories however, are centered on two particle descriptions, on exact diagonalizations of small samples, or mean field type of approximations ${ }^{6}$, i.e., exploring weak interacting limits. This contrasts with the recent band structure calculations ${ }^{7}$, which revealed the fact that the on-site Coulomb repulsion magnitude in these systems is actually relatively high, and may even reach $10 \mathrm{eV}$ causing strong correlation effects. Thus, exploring possibilities for other techniques compatible with strong correlation effects is needed for describing the origin of ferromagnetism in these systems.

In this Letter we present the result of an exact calculation applied to a multiband Hubbard model, by which it can be shown rigorously that ferromagnetic ground state appears well below quarter filling. A similar method was used previously ${ }^{\underline{8}}$ in polythiophene type structures in the high doping limit, i.e., well over the half filled case. In the present case however, working well below half filling, our results are much broader than the flat band approach $^{3,4}$ because we obtain ferromagnetism with sparse and even random presence of the local Coulomb repulsion, i.e., a Hubbard term is not required on every site.

Our analytical approach proceeds in three steps: the transformation of the Hamiltonian into positive semidefinite form, the construction of the ground state and the proof of their uniqueness. The technique is independent of the spatial dimension and does not require integrability of the model. The method was previously applied to construct the exact ground states for the Hubbard chains with different geometrical structures ${ }^{9}$ and even for two- -10 and three-dimensional periodic Anderson model ${ }^{11}$. Details of the method are described in Ref. ${ }^{12}$.

Following through with these steps, we start with i) transformation of a Hamiltonian into positive semidefinite form. A positive semidefinite operator $\hat{P}$ is defined as an operator which has only non-negative expectation values with all components $|\chi\rangle \in \mathcal{H}$ of the Hilbert space $\mathcal{H}$, i.e. $\langle\chi|\hat{P}| \chi\rangle \geq 0$. However, any Hamiltonian, $\hat{H}$, which describes a physical system is always bounded below and hence, can be written as $\hat{H}=\hat{P}+C$, where $\hat{P}$ a positive semidefinite and $C$ a scalar. It follows that the most general wave vector $\left|\Psi_{g}\right\rangle$ satisfying 
$\hat{P}\left|\Psi_{g}\right\rangle=0$ is the ground state wave vector of $\hat{H}$ with ground state energy $E_{g}=C$.

To show how straightforward the method is, we exemplify it's application with the twodimenisonal Hubbard model, given by the well-known Hamiltonian:

$$
\hat{H}=\sum_{\mathbf{i}, \sigma}\left(t_{x} \hat{c}_{\mathbf{i}+\mathbf{x}, \sigma}^{\dagger} \hat{c}_{\mathbf{i}, \sigma}+t_{y} \hat{c}_{\mathbf{i}+\mathbf{y}, \sigma}^{\dagger} \hat{c}_{\mathbf{i}, \sigma}+H . c\right)+\hat{H}_{U}, \quad \hat{H}_{U}=U \sum_{\mathbf{i}} \hat{n}_{\mathbf{i}, \sigma} \hat{n}_{\mathbf{i},-\sigma},
$$

on a square lattice with Bravais vectors $\mathbf{x}, \mathbf{y}$ and periodic boundary conditions. Here we used standard notations, where $\hat{c}_{\mathbf{j}, \sigma}^{\dagger}$ is the electron creation operator with spin projection $\sigma$, $\hat{n}_{\mathbf{j}, \sigma}=\hat{c}_{\mathbf{j}, \sigma}^{\dagger} \hat{c}_{\mathbf{j}, \sigma}$ is the number operator, $t_{x}$ and $t_{y}$ are the hopping matrix elements connecting nearest neighbour lattice sites in $x$ and $y$ directions, and $U>0$ represents the strength of the on-site Coulomb repulsion.

Per definition $\hat{H}_{U}$ is a positive semidefinite operator, however the total Hamiltonian, $\hat{H}$ is not. Hence, we perform a transformation on $\hat{H}$ to obtain one. For this, to each square, with coordinates $\mathbf{i}, \mathbf{i}+\mathbf{x}, \mathbf{i}+\mathbf{x}+\mathbf{y}, \mathbf{i}+\mathbf{y}$, we attach two block operators

$$
\hat{A}_{\mathbf{i}, \sigma}=a_{2} \hat{c}_{\mathbf{i}+\mathbf{x}, \sigma}+a_{3} \hat{c}_{\mathbf{i}+\mathbf{x}+\mathbf{y}, \sigma}+a_{4} \hat{c}_{\mathbf{i}+\mathbf{y}, \sigma}, \quad \hat{B}_{\mathbf{i}, \sigma}=b_{1} \hat{c}_{\mathbf{i}, \sigma}+b_{2} \hat{c}_{\mathbf{i}+\mathbf{x}, \sigma}+b_{4} \hat{c}_{\mathbf{i}+\mathbf{y}, \sigma},
$$

so as the starting Hamiltonian, Eq.(1), transforms into

$$
\hat{H}_{A B}=\sum_{\mathbf{i}, \sigma}\left(\hat{A}_{\mathbf{i}, \sigma}^{\dagger} \hat{A}_{\mathbf{i}, \sigma}+\hat{B}_{\mathbf{i}, \sigma}^{\dagger} \hat{B}_{\mathbf{i}, \sigma}\right)=\hat{H}-\hat{H}_{U}+q \hat{N},
$$

$\hat{P}=\hat{H}_{A B}+\hat{H}_{U}, \quad C=-q N$, with the number of electrons, $N$, fixed. The obtained $\hat{H}_{A B}$ is a positive semidefinite operator, and hence $\hat{P}=\hat{H}_{A B}+\hat{H}_{U}$ also. The only task left is to calculate the coefficients of the block operators, $\hat{A}_{\mathbf{i}, \sigma}$ and $\hat{B}_{\mathbf{i}, \sigma}$, for which the transformation into Eq.(3) gives:

$$
\begin{aligned}
& t_{x}=b_{2}^{*} b_{1}+a_{3}^{*} a_{4}, \quad t_{y}=b_{4}^{*} b_{1}+a_{3}^{*} a_{2}, \quad t_{y+x}=t_{y-x}=b_{4}^{*} b_{2}+a_{4}^{*} a_{2}=0, \\
& q=\left|b_{1}\right|^{2}+\left|b_{2}\right|^{2}+\left|b_{4}\right|^{2}+\left|a_{2}\right|^{2}+\left|a_{3}\right|^{2}+\left|a_{4}\right|^{2} .
\end{aligned}
$$

This system of equations represents the matching conditions. Obtaining a solution for these matching conditions implies a solution for the Hubbard Hamiltonian. This is usually not an easy task, as these equations are coupled, complex algebraic non-linear equations, but it can be done in some restricted $\hat{H}$ parameter space, e.g., see, Refs..$\underline{\underline{9}} \underline{\underline{11}}$ and even in disordered systems $\frac{13}{13}$.

Having a solution for the matching equations, we can easily go to the second step in our approach, namely ii) the construction of the ground state, i.e., $\left|\Psi_{g}\right\rangle$. The solution will depend 
on the structure of $\hat{P}$, however the most common case is when $\hat{P}$ operator contains terms of the form $\sum_{\mathbf{i}, \sigma} \hat{A}_{\mathbf{i}, \sigma}^{\dagger} \hat{A}_{\mathbf{i}, \sigma}, \sum_{\mathbf{i}, \sigma} \hat{B}_{\mathbf{i}, \sigma}^{\dagger} \hat{B}_{\mathbf{i}, \sigma}$. In these cases the ground state is constructed with the help of a block operator $\hat{C}_{\mathbf{j}, \sigma}^{\dagger}$ which anticommutes with $\hat{A}_{\mathbf{i}, \sigma}$ and $\hat{B}_{\mathbf{i}, \sigma}$, i.e., $\left\{\hat{A}_{\mathbf{i}, \sigma}, \hat{C}_{\mathbf{j}, \sigma^{\prime}}^{\dagger}\right\}=$ $\left\{\hat{B}_{\mathbf{i}, \sigma}, \hat{C}_{\mathbf{j}, \sigma^{\prime}}^{\dagger}\right\}=0$, for all possible values of all indices. Namely, if $|\chi\rangle=\prod_{\mathbf{i}, \sigma} \hat{C}_{\mathbf{i}, \sigma}^{\dagger}|0\rangle$ where $|0\rangle$ is the bare vacuum, then $\hat{H}_{A B}|\chi\rangle=0$. In order however, to obtain the ground state $\left|\Psi_{g}\right\rangle$ of $\hat{H}$ we need $|\chi\rangle$ to control all positive semidefinite operators in $\hat{P}$. In other words $|\chi\rangle$ has to be inserted in the kernel$\underline{\underline{14}}$ of all positive semidefinite operators existing in $\hat{P}$. This process is easily implemented by imposing some restrictions $(\mathbf{i}, \sigma) \in \mathcal{M}$ on the validity domain of $|\chi\rangle$, to be determined separately on model basis, after which the true ground state becomes $\left|\Psi_{g}\right\rangle=\prod_{(\mathbf{i}, \sigma) \in \mathcal{M}} \hat{C}_{\mathbf{i}, \sigma}^{\dagger}|0\rangle$.

The last step in our approach is iii) the proof of their uniqueness. For the most general case, when the ground state $\left|\Psi_{g}(m)\right\rangle$ is $M$ fold degenerate (i.e. $m=1,2, \ldots, M$ ), the proof of the uniqueness is done in two steps. In the first step, we prove that for all possible $m$ values $\left|\Psi_{g}(m)\right\rangle \in \operatorname{Ker}(\hat{P})$ is true. In the second step, we verify that any arbitrary wave vector $|\nu\rangle \in \operatorname{Ker}(\hat{P})$ can be expressed as a linear combination of the $\left|\Psi_{g}(m)\right\rangle$ terms, see Ref.11,12,15. In the non-degenerate case the steps are the same, but applied only to the $m=1$ ground state component.

In the following, we apply the above method to two cases of pentagon chains. First we analyse the pentagon chain without external links, see Fig. (11). This system is a conductor, a conjugated polymer of great interest which has not been analyzed yet with rigurous methods only way above the half filled concentration regime. Each pentagon cell contains four sites per cell. The cell defined at any site $\mathbf{i}$, see, the first cell of Fig. (11), has four adjacent sites at $\mathbf{i}+\mathbf{r}_{n}$, where $n=1,2,3,4$, and by convention $\mathbf{r}_{2}=0$. For a fixed $n$, the sites $\mathbf{i}+\mathbf{r}_{n}$ are belonging to the $n$-th sublattice. The on-site potentials and hopping transfer we used the notations shown on the second and third cell of the pentagon chain of Fig. (1).

With the above notations, the non-interacting part of the Hamiltonian becomes $\hat{H}_{0}=$ $\sum_{\sigma} \sum_{\mathbf{i}=1}^{N_{c}}\left\{\left[t\left(\hat{c}_{\mathbf{i}+\mathbf{r}_{1}, \sigma}^{\dagger} \hat{c}_{\mathbf{i}, \sigma}+\hat{c}_{\mathbf{i}+\mathbf{a}, \sigma}^{\dagger} \hat{c}_{\mathbf{i}+\mathbf{r}_{1}, \sigma}\right)+t_{n} \hat{c}_{\mathbf{i}+\mathbf{r}_{3}, \sigma}^{\dagger} \hat{c}_{\mathbf{i}+\mathbf{r}_{4}, \sigma}+t^{\prime}\left(\hat{c}_{\mathbf{i}, \sigma}^{\dagger} \hat{c}_{\mathbf{i}+\mathbf{r}_{3}, \sigma}+\hat{c}_{\mathbf{i}+\mathbf{r}_{4}, \sigma}^{\dagger} \hat{c}_{\mathbf{i}+\mathbf{a}, \sigma}\right)+H . c.\right]+\right.$ $\left.\left.\epsilon_{0}^{\prime} \hat{n}_{\mathbf{i}+\mathbf{r}_{1}, \sigma}++\epsilon_{1}^{\prime}\left(\hat{n}_{\mathbf{i}+\mathbf{r}_{3}, \sigma}+\hat{n}_{\mathbf{i}+\mathbf{r}_{4}, \sigma}\right)+\epsilon_{2}^{\prime} \hat{n}_{\mathbf{i}, \sigma}\right)\right\}$, where $N_{c}$ represents the number of cells. There are $4 N_{c}$ lattice sites in the system and $N$ electrons.

While, the interacting part of the Hamiltonian is $\hat{H}_{U}=\sum_{\mathbf{i}=1}^{N_{c}}\left[U_{0} \hat{n}_{\mathbf{i}+\mathbf{r}_{1}, \uparrow} \hat{n}_{\mathbf{i}+\mathbf{r}_{1}, \downarrow}+U_{1}\left(\hat{n}_{\mathbf{i}+\mathbf{r}_{3}, \uparrow} \hat{n}_{\mathbf{i}+\mathbf{r}_{3}, \downarrow}+\right.\right.$ $\left.\left.\hat{n}_{\mathbf{i}+\mathbf{r}_{4}, \uparrow} \hat{n}_{\mathbf{i}+\mathbf{r}_{4}, \downarrow}\right)+U_{2} \hat{n}_{\mathbf{i}, \uparrow} \hat{n}_{\mathbf{i}, \downarrow}\right]$, where, since in the positions $\mathbf{i}+\mathbf{r}_{1},\left(\mathbf{i}+\mathbf{r}_{3}, \mathbf{i}+\mathbf{r}_{4}\right)$, and $\mathbf{i}$ different type of atoms are potentially present holding in order the on-site one-particle potentials 


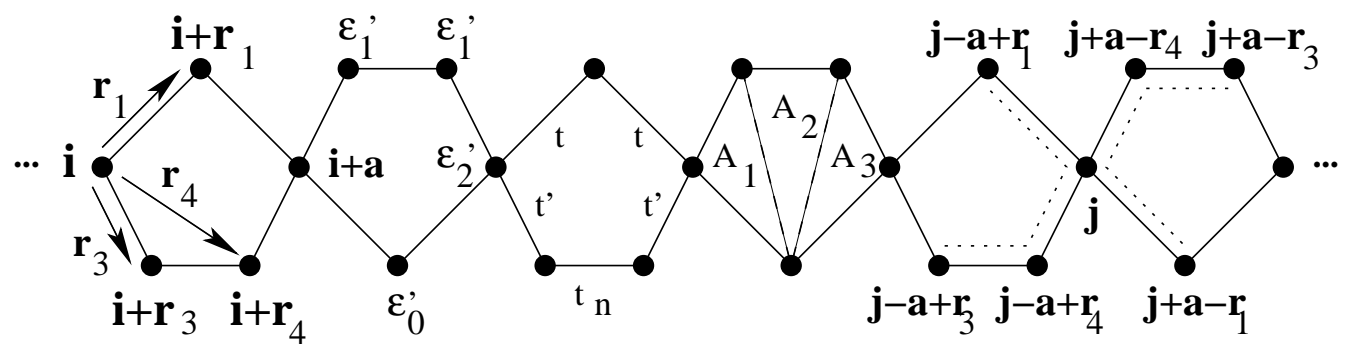

FIG. 1. The pentagon chain without external links. The first pentagon at site $\mathbf{i}$ shows the site coordinates; the on-site potentials and the hopping transfer mattrixes are shown on the second and third pentagons, respectively. The fourth pentagon depicts the triangular regions on which the block operators of Eq. (7) are defined. Finally, the last two pentagons present the sites (connected with dotted line as a guide to the eye) which contribute to the block operator $\hat{B}_{\mathbf{j}, \sigma}^{\dagger}$ defined in Eq. $(12)$.

$\epsilon_{0}^{\prime}, \epsilon_{1}^{\prime}$ and $\epsilon_{2}^{\prime}$, three different $U_{0}, U_{1}, U_{2}>0$ on-site Coulomb repulsion (Hubbard interaction) values are used. One has the Hubbard $U_{n}$ at the site where the on-site potential is $\epsilon_{n}^{\prime}$.

Hence, the total Hamiltonian will be simply $\hat{H}=\hat{H}_{0}+\hat{H}_{U}$ and using the technique previously detailed, see, Eqs. (2) - (41), the block operators are defined as:

$$
\begin{aligned}
& \hat{A}_{1, \mathbf{i}, \sigma}=a_{1,1} \hat{c}_{\mathbf{i}+\mathbf{r}_{1}, \sigma}+a_{1,2} \hat{c}_{\mathbf{i}, \sigma}+a_{1,3} \hat{c}_{\mathbf{i}+\mathbf{r}_{3}, \sigma}, \\
& \hat{A}_{2, \mathbf{i}, \sigma}=a_{2,1} \hat{c}_{\mathbf{i}+\mathbf{r}_{1}, \sigma}+a_{2,3} \hat{c}_{\mathbf{i}+\mathbf{r}_{3}, \sigma}+a_{2,4} \hat{c}_{\mathbf{i}+\mathbf{r}_{4}, \sigma}, \\
& \hat{A}_{3, \mathbf{i}, \sigma}=a_{3,1} \hat{c}_{\mathbf{i}+\mathbf{r}_{1}, \sigma}+a_{3,4} \hat{c}_{\mathbf{i}+\mathbf{r}_{4}, \sigma}+a_{3,5} \hat{c}_{\mathbf{i}+\mathbf{a}, \sigma} .
\end{aligned}
$$

These operators $\operatorname{span}^{16}$ a pentagon cell as depicted in the fourth cell of Fig.(1). Using periodic boundary conditions $\hat{H}_{0}$ transfroms into:

$$
\hat{H}_{0}=\sum_{\sigma} \sum_{\mathbf{i}=1}^{N_{c}} \sum_{m=1}^{3} \hat{A}_{m, \mathbf{i}, \sigma}^{\dagger} \hat{A}_{m, \mathbf{i}, \sigma} .
$$

We are interested to find the ground state solution well below quarter filling, hence we work in the condition $N \leq N_{c}$. For the solution of the matching conditions, for real hopping 
matrix elements and conditions $t_{n}>0, \epsilon_{1}^{\prime}-t_{n}>0$ we obtained:

$$
\begin{aligned}
& a_{1,1}=e^{i \phi_{1}}\left|a_{1,1}\right|, \quad a_{1,2}=e^{i \phi_{1}} \frac{t}{\left|a_{1,1}\right|}, \quad a_{1,3}=e^{i \phi_{1}} \frac{t^{\prime}}{t}\left|a_{1,1}\right|, \\
& a_{2,1}=e^{i \phi_{2}}\left|a_{2,1}\right|, \quad a_{2,3}=-e^{i \phi_{2}} \frac{t^{\prime}}{t} \frac{\left|a_{1,1}\right|^{2}}{\left|a_{2,1}\right|}, \quad a_{2,4}=-e^{i \phi_{2}} \frac{t t_{n}}{t^{\prime}} \frac{\left|a_{2,1}\right|}{\left|a_{1,1}\right|^{2}} \\
& a_{3,1}=e^{i \phi_{3}}\left|a_{3,1}\right|, \quad a_{3,4}=e^{i \phi_{3}} \frac{t^{\prime}}{t}\left|a_{3,1}\right|, \quad a_{3,5}=e^{i \phi_{3}} \frac{t}{\left|a_{3,1}\right|},
\end{aligned}
$$

where $\phi_{m}, m=1,2,3$ are arbitrary phases. In Eqs. (7) the Hamiltonian parameters $t, t^{\prime}, t_{n}, \epsilon_{1}^{\prime}$ can be arbitrary chosen, while $\epsilon_{0}^{\prime}, \epsilon_{2}^{\prime}$ are given by the conditions $\epsilon_{0}^{\prime}=\left[t^{2} /\left(t^{\prime 2} t_{n}\right)\right]\left(\epsilon_{1}^{\prime 2}-t_{n}^{2}\right)$, and $\epsilon_{2}^{\prime}=2 t^{\prime 2} /\left(\epsilon_{1}^{\prime}-t_{n}\right)$. These last two conditions provide the lowest flat band of the band structure.

Since $\hat{H}_{0}$ has the simple expression (6) , we look for the ground state wave function in the form

$$
\left|\Psi_{g}\right\rangle=\prod_{\mathbf{i}=1}^{N \leq N_{c}} \hat{B}_{\mathbf{i}, \sigma_{\mathbf{i}}}^{\dagger}|0\rangle,
$$

where $|0\rangle$ is the bare vacuum, and $\hat{B}_{\mathbf{i}, \sigma_{\mathbf{i}}}^{\dagger}$ satisfies for all $n=1,2,3$ the relation

$$
\left\{\hat{A}_{n, \mathbf{i}, \sigma}, \hat{B}_{\mathbf{i}^{\prime}, \sigma_{\mathbf{i}^{\prime}}^{\prime}}^{\dagger}\right\}=0
$$

where $\mathbf{i}, \mathbf{i}^{\prime}, \sigma, \sigma_{\mathbf{i}^{\prime}}^{\prime}$ are arbitrary. Since only one type of canonical Fermi operator is defined on each site, Eq. (8) is true if the $\hat{B}_{\mathbf{i}^{\prime}, \sigma_{\mathbf{i}^{\prime}}^{\prime}}^{\dagger}$ operators do not overlap, or the neighbouring operators overlap at least on one site.

The first case, when the $\hat{B}_{\mathbf{i}^{\prime}, \sigma_{\mathbf{i}^{\prime}}^{\prime}}^{\dagger}$ operators do not overlap, would mean a localized and paramagnetic ground state of the general form $\hat{B}_{\mathbf{i}, \sigma}^{\dagger}=x_{1} \hat{c}_{\mathbf{i}+\mathbf{r}_{1}, \sigma}^{\dagger}+x_{3} \hat{c}_{\mathbf{i}+\mathbf{r}_{3}, \sigma}^{\dagger}+x_{4} \hat{c}_{\mathbf{i}+\mathbf{r}_{4}, \sigma}^{\dagger}$. However, there isn't any value of $x_{1}, x_{2}, x_{3}$, except $x_{1}=x_{3}=x_{4}=0$, which would satisfy Eq. (9), hence there is no solution in this case.

To search for a solution in the second case, i.e., when the $\hat{B}_{\mathbf{i}^{\prime}, \sigma_{\mathbf{i}^{\prime}}^{\prime}}^{\dagger}$ operators overlap, we define $\hat{B}_{\mathbf{i}, \sigma}^{\dagger}$ as shown on the last two cells of Fig. (1), namely:

$$
\begin{aligned}
\hat{B}_{\mathbf{i}, \sigma}^{\dagger} & =x_{1} \hat{c}_{\mathbf{i}+\mathbf{r}_{1}, \sigma}^{\dagger}+x_{2} \hat{c}_{\mathbf{i}, \sigma}^{\dagger}+x_{3} \hat{c}_{\mathbf{i}+\mathbf{r}_{3}, \sigma}^{\dagger}+x_{4} \hat{c}_{\mathbf{i}+\mathbf{r}_{4}, \sigma}^{\dagger} \\
& +y_{1} \hat{c}_{\mathbf{i}-\mathbf{a}+\mathbf{r}_{1}, \sigma}^{\dagger}+y_{3} \hat{c}_{\mathbf{i}-\mathbf{a}+\mathbf{r}_{3}, \sigma}^{\dagger}+y_{4} \hat{c}_{\mathbf{i}-\mathbf{a}+\mathbf{r}_{4}, \sigma}^{\dagger}
\end{aligned}
$$

and the solution to (9) is:

$$
\begin{aligned}
& x_{4}=-\frac{t}{t^{\prime}} x_{1}, \quad x_{3}=\frac{t \epsilon_{1}^{\prime}}{t^{\prime} t_{n}} x_{1}, \quad x_{2}=-\frac{t}{t^{\prime 2} t_{n}}\left(\epsilon_{1}^{\prime 2}-t_{n}^{2}\right) x_{1}, \\
& y_{1}=x_{1}, \quad y_{3}=x_{4}=-\frac{t}{t^{\prime}} x_{1}, \quad y_{4}=x_{3}=\frac{t \epsilon_{1}^{\prime}}{t^{\prime} t_{n}} x_{1} .
\end{aligned}
$$


Consequently, the $\hat{B}_{\mathbf{i}, \sigma}^{\dagger}$ operator becomes

$$
\begin{aligned}
\hat{B}_{\mathbf{i}, \sigma}^{\dagger} & =x_{1}\left[-\frac{t\left(\epsilon_{1}^{\prime 2}-t_{n}^{2}\right)}{t^{\prime 2} t_{n}} \hat{c}_{\mathbf{i}, \sigma}^{\dagger}+\left(\hat{c}_{\mathbf{i}+\mathbf{r}_{1, \sigma}}^{\dagger}+\hat{c}_{\mathbf{i}-\mathbf{a}+\mathbf{r}_{1}, \sigma}^{\dagger}\right)+\frac{t \epsilon_{1}^{\prime}}{t^{\prime} t_{n}}\left(\hat{c}_{\mathbf{i}+\mathbf{r}_{3}, \sigma}^{\dagger}+\hat{c}_{\mathbf{i}-\mathbf{a}+\mathbf{r}_{3}, \sigma}^{\dagger}\right)\right. \\
& \left.-\frac{t}{t^{\prime}}\left(\hat{c}_{\mathbf{i}+\mathbf{r}_{4}, \sigma}^{\dagger}+\hat{c}_{\mathbf{i}-\mathbf{a}+\mathbf{r}_{3}, \sigma}^{\dagger}\right)\right] .
\end{aligned}
$$

The (unnormalized) ground state wave function at $1 / 8$ filling (e.g. $N=N_{c}$ ) becomes a saturated ferromagnet

$$
\left|\Psi_{g}\right\rangle=\prod_{\mathbf{i}=1}^{N_{c}} \hat{B}_{\mathbf{i}, \sigma}^{\dagger}|0\rangle
$$

where $\sigma$ is fixed. Below $1 / 8$ filling the block operator $\hat{B}_{\mathbf{i}, \sigma}^{\dagger}$ is still given in Eq. (12), and the ground state will have the (8) form. But, a geometrical degeneracy occurs: only overlaping $\hat{B}_{\mathbf{i}, \sigma}^{\dagger}$ operators will have the same spin index. Hence, the ground state will be composed from ferromagnetic clusters which if don't overlap, will have arbitrary spin orientations. We should also point out that Eq. (13) corresponds to the half filled lower flat band. The obtained solution is true for arbitrary large $U_{0}, U_{1}, U_{2}>0$ Hubbard terms.

Next, we analyse the second model of a pentagon chain, namely the pentagon chain with external links and antennas, see Fig. (2). This chain is also a conductor, and we are going to show in the following that the obtained results are qualitatively the same as in the previous case. The new pentagon chain, with external links and antennas connected to the pentagons, is shown in Fig. (22). The cell now contains six sites and consequently, there will be six sublattices in the system. The cell defined at any site $\mathbf{i}$, see, the first cell of Fig. (2) has six adjacent sites at $\mathbf{i}+\mathbf{r}_{n}$, where now $n=1,2, \ldots, 6$, and $\mathbf{r}_{3}=0$ by convention. For a fixed $n$, the sites $\mathbf{i}+\mathbf{r}_{n}$ are belonging to the $n$-th sublattice.

With the on-site potentials and hopping matrix elements defined on the second and third cell of Fig. (2), the starting Hamiltonian $\hat{H}=\hat{H}_{0}+\hat{H}_{U}$ becomes: $\hat{H}_{0}=\sum_{\sigma} \sum_{\mathbf{i}=1}^{N_{c}}\left\{\left[t_{f} \hat{c}_{\mathbf{i}+\mathbf{r}_{1}, \sigma}^{\dagger} \hat{c}_{\mathbf{i}+\mathbf{r}_{2}, \sigma}+\right.\right.$ $\left.t_{c} \hat{c}_{\mathbf{i}+\mathbf{r}_{6}, \sigma}^{\dagger} \hat{c}_{\mathbf{i}+\mathbf{a}, \sigma}+t_{n} \hat{c}_{\mathbf{i}+\mathbf{r}_{4}, \sigma}^{\dagger} \hat{c}_{\mathbf{i}+\mathbf{r}_{5}, \sigma}+t\left(\hat{c}_{\mathbf{i}+\mathbf{r}_{2}, \sigma}^{\dagger} \hat{c}_{\mathbf{i}, \sigma}+\hat{c}_{\mathbf{i}, \sigma}^{\dagger} \hat{c}_{\mathbf{i}+\mathbf{r}_{4}, \sigma}+\hat{c}_{\mathbf{i}+\mathbf{r}_{5}, \sigma}^{\dagger} \hat{c}_{\mathbf{i}+\mathbf{r}_{6}, \sigma}+\hat{c}_{\mathbf{i}+\mathbf{r}_{6}, \sigma}^{\dagger} \hat{\sigma}_{\mathbf{i}+\mathbf{r}_{2}, \sigma}\right)+H . c.\right]+$ $\left.\epsilon_{0}^{\prime}\left(\hat{n}_{\mathbf{i}+\mathbf{r}_{1}, \sigma}+\hat{n}_{\mathbf{i}+\mathbf{r}_{2}, \sigma}\right)+\epsilon_{1}^{\prime}\left(\hat{n}_{\mathbf{i}+\mathbf{r}_{4}, \sigma}+\hat{n}_{\mathbf{i}+\mathbf{r}_{5}, \sigma}\right)+\epsilon_{2}^{\prime}\left(\hat{n}_{\mathbf{i}, \sigma}+\hat{n}_{\mathbf{i}+\mathbf{r}_{6}, \sigma}\right)\right\}$, while the interacting part of the Hamiltonian is now $\hat{H}_{U}=\sum_{\mathbf{i}=1}^{N_{c}}\left[U_{0}\left(\hat{n}_{\mathbf{i}+\mathbf{r}_{1}, \uparrow} \hat{n}_{\mathbf{i}+\mathbf{r}_{1}, \downarrow}+\hat{n}_{\mathbf{i}+\mathbf{r}_{2}, \uparrow} \hat{n}_{\mathbf{i}+\mathbf{r}_{2}, \downarrow}\right)+U_{1}\left(\hat{n}_{\mathbf{i}+\mathbf{r}_{4}, \uparrow} \hat{n}_{\mathbf{i}+\mathbf{r}_{4}, \downarrow}+\right.\right.$ $\left.\left.\hat{n}_{\mathbf{i}+\mathbf{r}_{5}, \uparrow} \hat{n}_{\mathbf{i}+\mathbf{r}_{5}, \downarrow}\right)+U_{2}\left(\hat{n}_{\mathbf{i}, \uparrow} \hat{n}_{\mathbf{i}, \downarrow}+\hat{n}_{\mathbf{i}+\mathbf{r}_{6}, \uparrow} \hat{n}_{\mathbf{i}+\mathbf{r}_{6}, \downarrow}\right)\right]$. In the interacting part of the Hamiltonian, since in positions $\left(\mathbf{i}+\mathbf{r}_{1}, \mathbf{i}+\mathbf{r}_{2}\right),\left(\mathbf{i}+\mathbf{r}_{4}, \mathbf{i}+\mathbf{r}_{5}\right)$, and $\left(\mathbf{i}, \mathbf{i}+\mathbf{r}_{6}\right)$ different type of atoms are present, three are three different $U_{0}, U_{1}, U_{2}>0$ local Coulomb repulsion values. Also, the number of lattice sites on the chain is $6 N_{c}$, and the number of electrons is $N$. 


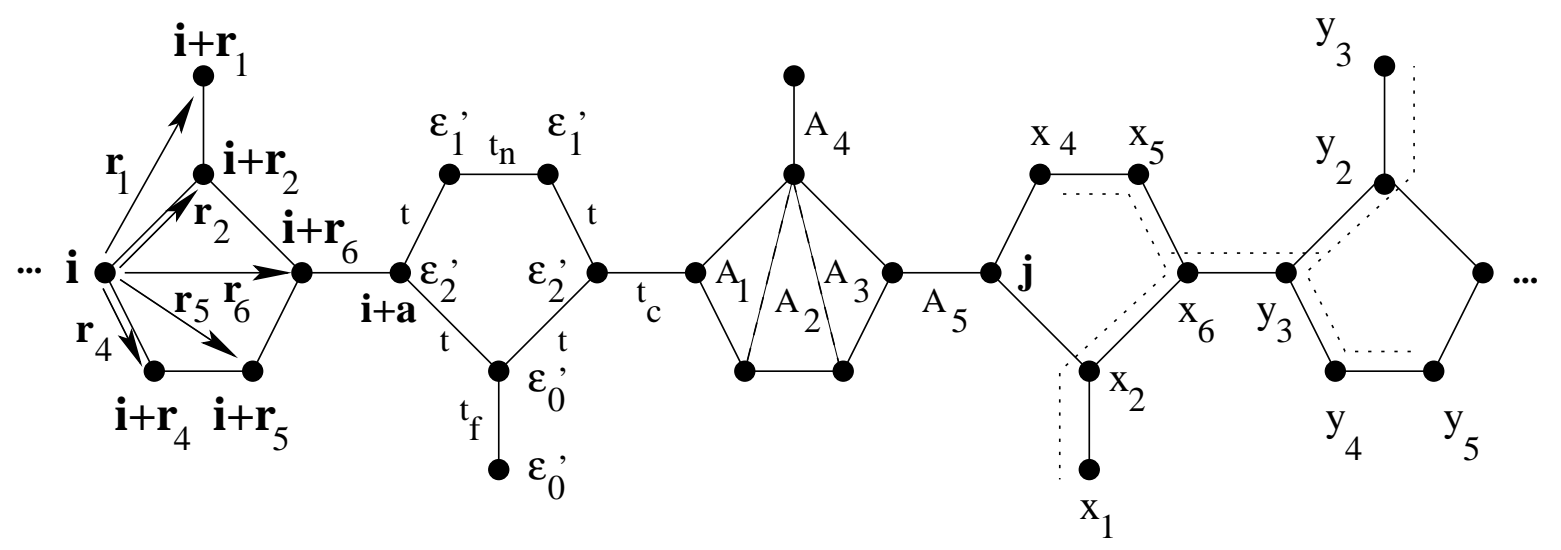

FIG. 2. The pentagon chain with external links and antennas. The first pentagon at site $\mathbf{i}$ shows the site coordinates; the on-site potentials and the hopping transfermattrixes are shown on the second pentagon. The third pentagon depicts the five regions (three triangular and two bond domains) on which the block operators from Eq. (18) are defined. Finally, the last two pentagons present the sites (connected with dotted line as a guide to the eye) which contribute to the block operator $\hat{B}_{\mathbf{j}, \sigma}^{\text {dagger }}$ defined in Eq. (23). The coefficients of each individual site contributions $\left(x_{i}, y_{i}\right)$ are also shown.

Using the same approach as for the previously analysed case, we obtain for the block operator $\hat{B}_{\mathbf{i}, \sigma}^{\dagger}$ :

$$
\begin{aligned}
\hat{B}_{\mathbf{i}, \sigma}^{\dagger} & =x_{1}\left[\hat{c}_{\mathbf{i}+\mathbf{r}_{1}, \sigma}^{\dagger}-\frac{\epsilon_{0}^{\prime}}{t_{f}} \hat{c}_{\mathbf{i}+\mathbf{r}_{2}, \sigma}^{\dagger}+\frac{\epsilon_{0}^{\prime}}{t_{f}} \hat{c}_{\mathbf{i}+\mathbf{r}_{4}, \sigma}^{\dagger}-\frac{\epsilon_{0}^{\prime} \epsilon_{1}^{\prime}}{t_{f} t_{n}} \hat{c}_{\mathbf{i}+\mathbf{r}_{5}, \sigma}^{\dagger}+\frac{\epsilon_{0}^{\prime}\left(\epsilon_{1}^{\prime 2}-t_{n}^{2}\right)}{t t_{f} t_{n}} \hat{c}_{\mathbf{i}+\mathbf{r}_{6}, \sigma}^{\dagger}\right. \\
& -\left(\epsilon_{1}^{\prime}-t_{n}\right) \operatorname{sign}\left(t_{c}\right)\left(\hat{c}_{\mathbf{i}+\mathbf{a}+\mathbf{r}_{1}, \sigma}^{\dagger}-\frac{\epsilon_{0}^{\prime}}{t_{f}} \hat{c}_{\mathbf{i}+\mathbf{a}+\mathbf{r}_{2}, \sigma}^{\dagger}+\frac{\epsilon_{0}^{\prime}}{t_{f}} \hat{c}_{\mathbf{i}+\mathbf{a}+\mathbf{r}_{5}, \sigma}^{\dagger}\right. \\
& \left.\left.-\frac{\epsilon_{0}^{\prime} \epsilon_{1}^{\prime}}{t_{f} t_{n}} \hat{c}_{\mathbf{i}+\mathbf{a}+\mathbf{r}_{4}, \sigma}^{\dagger}+\frac{\epsilon_{0}^{\prime}\left(\epsilon_{1}^{\prime 2}-t_{n}^{2}\right)}{t t_{f} t_{n}} \hat{c}_{\mathbf{i}+\mathbf{a}, \sigma}^{\dagger}\right)\right] .
\end{aligned}
$$

The (unnormalized) ground state wave function at 1/12 filling (e.g. $N=N_{c}$ ) becomes a saturated ferromagnet

$$
\left|\Psi_{g}\right\rangle=\prod_{\mathbf{i}=1}^{N_{c}} \hat{B}_{\mathbf{i}, \sigma}^{\dagger}|0\rangle,
$$

where $\sigma$ is fixed. Below 1/12 filling the expression of $\hat{B}_{\mathbf{i}, \sigma}^{\dagger}$ remains as given in Eq. (14), but in (15) a geometrical degeneracy occurs, only overlaping $\hat{B}_{\mathbf{i}, \sigma}^{\dagger}$ operators will have the same spin 
index, and the ground state will be constructed from ferromagnetic clusters which if not in contact, will have arbitrary spin orientation. The ground state given by Eq. (15) corresponds to a half filled lowest flat band. The obtained solution however, occurs for arbitrary large $U_{0}, U_{1}, U_{2}>0$ Hubbard interaction terms. Similar situations for other compounds have been intensively analyzed in literature ${ }^{22-24}$.

In summary, by employing a rigorous analytical method we have constructed exact ground states for multiorbital pentagon Hubbard chains. The ferromagnetism what we found well below half filling originate from the multi-orbital polygon chains which yield dispersionless band in the presence of site-dependent Coulomb intercation. $N$ dependent ground states we have obtained for $N \leq N_{c}$, and the system is conducting for $N<N_{c}$. At $N=N_{c}$, the ferromagnetism emerges since in the ground state wave vector all contributing terms have the same fixed spin projection. The proof of the uniqueness of our results can be made along the lines of Refs. $\stackrel{12,15}{ }$.

Organic ferromagnets have attracted much attention as a challenging target. In particular, organic magnets consisting entirely of non-magnetic elements is of fundamental as well as practical interest. Ordinary ferromagnets consist of magnetic elements and even onedimensionals models which exhibit ferromagnetism exploit electrons in $d$ or $f$ orbitals. In the presence of strong interaction, for example such in the Kondo lattice case, the $f$ electrons are responsible for ferromagnetism which, as it was shown in Refs. 17 using non-Abelian density matrix renormalization group $\frac{18}{}$, order due to scattering with the conduction electrons. Since hopping is energetically most favorable for conductions electrons which preserver their spins, called coherent hopping, this tends to align the localized $f$ electron spins 17 .

But, in the cases analysed in this Letter only non-magnetic elements are present in the pentagon chain. We can rightfully ask the question how magnetism can arise in these systems? The answer to this question is that the Coulomb intercations are capable of turning itinerant system into a ferromagnetic phase in an extended parameter region. The magnetism arises as an effect of the electron-electron repulsion when the adjacent block operators which yield the ground state wave vector overlap and intuitively the spin has to align to lower the repulsion energy due to Pauli's principle.

Continuing the above agurment, due to the overlaping adjacent block operators, in our model we do not even need all sites to be interacting, it is enoguh to have merely one site to be intercating in each cell. To show this, let us consider first the pentagon chain wihtout 
external links. The sites contributing to the block operators of the ground state wave vector are shown in Fig. (1) with dotted lines (last two pentagon of the figure). Consequently, at $N=N_{c}$ number of electrons, ferromagnetism will appear even if there is only one site in each pentagon with non zero local Coulomb repulsion, namely one of the sites with coordinates $\mathbf{r}_{1}, \mathbf{r}_{3}, \mathbf{r}_{4}$. On these sites, even a random distribution of one local Coulomb repulsion on each cell yields ferromagnetism. We note that in this case $75 \%$ of sites are non-interacting (three sites from four in each cell), i.e. without Hubbard interaction.

The same is true for the case of pentagon chains with external links. If one site per pentagon has a Hubbard $\mathrm{U}$ attached to it, in between sites with coordinates $\mathbf{r}_{1}, \mathbf{r}_{2}, \mathbf{r}_{4}, \mathbf{r}_{5}, \mathbf{r}_{6}$, see, Fig. (2). In this case $83.3 \%$ of sites are non-interacting (five sites out of six in each cell). This shows that surprizingly, the complete absence of magnetic atoms with sparse and even random presence of the local Coulomb repulsion can lead to ferromagnetism. This underlines that the conditions in which we obtained ferromagnetism are much broader than those fixed by flat-band ferromagnetism, where on every site of the system $U>0$ is required $\underline{25}$. Hence, the obtained solutions point to a new route for the design of ferromagnetic chain polymners.

Regarding the experimental observation of ferromagnetism, we have to point out that the required electron doping of the pentagon chains can be achieved $\underline{\underline{19}}$ by changing the Fermi level by selecting appropriate side groups or by field-effect doping in a double-layer transisitor structure ${ }^{20}$. Indeed, depending on the applied doping levels pentagon polymers can be turned $\underline{21}$ into ferromagnets, spin glasses or simple paramagnetic polymers.

\section{Acknowledgements}

(1) For M. Gulácsi this research was realized in the frames of TAMOP 4.2.4. A/2-11-12012-0001 "National Excellence Program - Elaborating and operating an inland student and researcher personal support system". The project was subsidized by the European Union and co-financed by the European Social Fund.

(2) Zs. Gulácsi kindly acknowledges financial support provided by Alexander von Humboldt Foundation, OTKA-K-100288 (Hungarian Research Funds for Basic Research) and TAMOP 4.2.2/A-11/1/KONV-2012-0036 (co-financed by EU and European Social Fund). 
1 A. A. Correa, et al., Synth. Met. 121 (2001) p. 1836; O. R. Nascimento, et al., Phys. Rev. B67 (2003) 144422; F. R. de Paula, et al., 320 (2008) p. 193; S. Majumdar, et al., arXiv: 0905.2021.

2 Y. Suwa, et al., Phys. Rev. B68 (2003) p. 174419; R. Arita, et al., Phys. Rev. Lett. 88 (2002) p. 127202; ibid., Phys. Rev. B68 (2003) p. 140403.

3 A. Mielke and H. Tasaki, Commun. Math. Phys. 158 (1993) p. 341.

4 A. Mielke, Phys. Lett. A174 (1993) p. 443; J. Phys. A: Math. Gen 32 (1999) p. 8411.

5 Y. Suwa, et al., Phys. Rev. B82 (2010) p. 235127.

6 See, for example M. Gulacsi and Z. Gulacsi, Phys. Rev. B33 (1986) p. 6147; Z. Gulacsi and M. Gulacsi, Phys. Rev. B36 (1987) p. 699; and Z. Gulacsi, M. Gulacsi and I. Pop, Phys. Rev. B37 (1988) p. 2247.

7 T. O. Wehling, et al., Phys. Rev. Lett. 106 (2011) p. 236805.

8 Z. Gulacsi, A. Kampf and D. Vollhardt, Phys. Rev. Lett. 105 (2010) p. 266403.

9 Z. Gulacsi, A. Kampf and D. Vollhardt, Phys. Rev. Lett. 99 (2007) p. 026404; Z. Gulacsi and I. Orlik, Jour. Phys. A34 (2001) L359.

10 I. Orlik and Z. Gulacsi, Phil. Mag. Lett. 78 (1998) p. 177; P. Gurin and Z. Gulacsi, Phys. Rev. B64 (2001) p. 045118; Z. Gulacsi and M. Gulacsi, Phys. Rev. B73 (2006) p. 014514.

11 Z. Gulacsi and D. Vollhardt, Phys. Rev. Lett. 91 (2003) p. 186401; ibid., Phys. Rev. B72 (2005) p. 075130.

12 Z. Gulacsi, A. Kampf and D. Vollhardt, Prog. Theor. Phys. Suppl. 176 (2008) p. 1.

13 Z. Gulacsi, Phys. Rev. B69 (2004) p. 054204; Phys. Rev. B66 (2002) p. 165109; Eur. Phys. Jour. B30 (2002) p. 295.

14 The kernel $\operatorname{Ker}(\hat{O})$ of an arbitrary operator $\hat{O}$ is a Hilbert subspace containing all wave vectors $|\phi\rangle$ with the property $\hat{O}|\phi\rangle=0$.

15 Z. Gulacsi, Int. J. Mod. Phys. B27 (2013) p. 1330009.

16 M. Gulacsi, Phil. Mag. B76 (1997) p. 731; M. Gulacsi, H. van Beijeren and A. C. Levi, Phys. Rev. E47 (1993) p. 2473.

17 I. P. McCulloch, et al., J. Low Temp. Phys. 117 (1999) p. 323; I. P. McCulloch, et al., Phil. Mag. Lett. 81 (2001) p. 869; I. P. McCulloch, et al., Phys. Rev. B65 (2002) p. 052410.

18 I. P. McCulloch and M. Gulacsi, Aust. J. Phys. 53 (2000) p. 597; ibid., Phil. Mag. Lett. 81 
(2001) p. 447; ibid., Europhys. Lett 57 (2002) p. 852.

19 A. Opitz, et al., New J. Phys. 10 (2008) p. 065006.

20 K. Ueno, et al., Nat. Mater. 7 (2008) p. 855; H. Yuan, et al., Adv. Funct. Mater. 19 (2009)p. 1046.

21 M. J. Panzer, et al., Appl. Phys. Lett. 86 (2005) p. 022104; M. J. Panzer and C. D. Frisbie, J. Am. Chem. Soc. 127 (2005) p. 6960.

22 S. Capponi, O. Derzhko, A. Honecker, et al., Phys. Rev. B88 (2013) p. 144416.

23 O. Derzhko, J. Richter, O. Krupnitska, et al., Phys. Rev. B88 (2013) p. 094426.

24 M. Maksymenko, A. Honecker, R. Moessner, et al., Phys. Rev. Lett. 109 (2012) p.096404.

25 In order to present this statement in mathematical terms, we mention that for example in the first paper of Ref.[4] (Phys.Lett A174,443(1993)), if 75-80\% of the sites are non-interacting, Eq.(18) on pg. 445 is no more valid, hence the proof started on pg.444 is no more correct. 